\title{
The U-box ligase carboxyl-terminus of Hsc 70-interacting protein ubiquitylates Epsin
}

\author{
Yoav E. Timsit ${ }^{\mathrm{a}}$, Stephanie L.H. Miller ${ }^{\mathrm{a}, \mathrm{b}}$, Robert P. Mohney ${ }^{\mathrm{a}, 1}$, John P. O'Bryan ${ }^{\mathrm{a}, *}$ \\ a Laboratory of Signal Transduction, National Institute of Environmental Health Sciences, National Institutes of Health, \\ Department of Health and Human Services, Research Triangle Park, NC 27709, USA \\ ${ }^{\mathrm{b}}$ Department of Biomedical Engineering, School of Medicine, University of North Carolina at Chapel Hill, Chapel Hill, NC 27599, USA
}

Received 5 January 2005

Available online 13 January 2005

\begin{abstract}
Epsin is an endocytic adaptor protein involved in the regulation of clathrin-dependent endocytosis. We and others have demonstrated that Epsin is ubiquitylated in cells and requires its ubiquitin interacting motifs (UIMs) for this modification. To further elucidate the mechanism of Epsin ubiquitylation, we initiated studies to identify the E3 ligase(s) that modifies Epsin. In this study, we discovered that the U-box ubiquitin ligase carboxyl-terminus of Hsc70 interacting protein (CHIP) ubiquitylated Epsin. Using an in vitro ubiquitylation assay, we demonstrate that CHIP specifically ubiquitylated Epsin in a UIM-dependent manner. Furthermore, overexpression of CHIP in cells increased Epsin ubiquitylation also in a UIM-dependent manner. Together, these data provide evidence that CHIP functions to ubiquitylate the endocytic protein Epsin.

Published by Elsevier Inc.
\end{abstract}

Keywords: Endocytosis; Ubiquitylation; Trafficking; UIMs

Clathrin-dependent endocytosis involves a variety of modular domain-containing proteins that function at various steps in the clathrin-mediated internalization pathway [1,2]. These proteins include Eps15 and Epsin (Eps15 interactor) that are recruited in conjunction with AP-2, dynamin, clathrin, and various other proteins to regions of the cell membrane that undergo internalization. Epsin acts as a molecular bridge between the membrane and endocytic adaptors by virtue of its $\mathrm{NH}_{2}$-terminal, phosphoinositol-binding ENTH

\footnotetext{
Abbreviations: GST, glutathione-S-transferase; ENTH, Epsin $\mathrm{NH}_{2}$-terminal homology domain; $\mathrm{EGF}(\mathrm{R})$, epidermal growth factor (receptor); UIMs, ubiquitin-interacting motifs; Ub, ubiquitin; HA, hemagglutinin; CHIP, carboxyl-terminus of $\mathrm{Hsc} 70$ interacting protein; DUB, deubiquitylating enzyme.

* Corresponding author. Fax: +1919541 1898.

E-mail addresses: obryan@niehs.nih.gov (J.P. O’Bryan).

${ }^{1}$ Present address: Amphora Discovery Corporation, P.O. Box 12169, Research Triangle Park, NC 27709, USA.
}

domain, and $\mathrm{COOH}$-terminal NPF motifs that interact with Eps15 and intersectin $[3,4]$. These protein complexes signal the recruitment of the remaining components of the internalization pathway, such as AP-2, clathrin, synaptojanin, and dynamin, that function in the maturation of a clathrin-coated pit into an intracellular clathrin-coated vesicle (CCV) [5].

Over the past several years, new insights have been obtained into the mechanisms by which clathrin-dependent endocytosis is regulated in cells. Evidence has accumulated for the importance of ubiquitin in endocytosis, with much attention focused on the ubiquitylation and/ or ubiquitin-binding activities of endocytic adaptor proteins (reviewed in [6]). In the case of the endocytic adaptor Epsin, genetic studies have revealed the contribution of the ubiquitylation pathway for photoreceptor development in Drosophila. Namely, loss-of-function mutations in the Drosophila deubiquitylating enzyme Fat facets (Faf) produce abnormal eye phenotypes that are 
attributed to aberrant endocytosis due to the inability of Faf to deubiquitylate the Drosophila Epsin, liquid facets (Lqf) $[7,8]$. Thus, the function of Epsin in endocytosis appears to be intimately linked to the ubiquitylation pathway. In addition, Epsin is predominantly monoubiquitylated in cells [9-11], a modification observed with other proteins involved in endocytosis and/or signaling [9,11-14]. Taken together, these data suggest that components of the endocytic machinery require covalent modification with ubiquitin, and that this modification predominantly involves the attachment of a single ubiquitin moiety. In the case of Epsin, recent evidence suggests that monoubiquitylation of Epsin regulates its interaction with membranes [15]. However, the mechanism(s) by which monoubiquitylated forms of Epsin are produced remain unclear.

In order to address these issues, we and others have evaluated the structural determinants of Epsin and Eps15 that signal their ubiquitylation. A novel motif termed the ubiquitin-interacting motif (UIM) was identified in Epsin and Eps15, and found to be critical for the ubiquitylation of these proteins [10,11]. Epsin, as well as several additional UIM-containing proteins, was ubiquitylated in a UIM-dependent manner, producing a predominant monoubiquitylated species that was not subjected to proteosomal degradation $[10,11]$. In addition, UIMs from various endocytic proteins confer differences in the ability of these proteins to be ubiquitylated and to non-covalently bind ubiquitin [9]. Collectively, these findings indicate that the UIM represents a signal that dictates the ability of a protein to be ubiquitylated. Identifying the E3 ubiquitin ligase(s) that catalyzes ubiquitylation of UIM-containing proteins such as Epsin will undoubtedly facilitate a more detailed understanding of the biological importance and function of ubiquitylated endocytic adaptor proteins in endocytosis.

In the present study, we observed that a $70-\mathrm{kDa}$ protein co-precipitated with the UIMs of Epsin. Mass-spectrometry analysis confirmed the identity of this protein as heat shock protein $70(\mathrm{Hsc} / \mathrm{Hsp} 70)$. Since Hsc70 interacts with the U-box E3 ligase carboxyl-terminus of $\mathrm{Hsc} 70 / \mathrm{Hsp} 70$ interacting protein (CHIP), we propose a model for Epsin ubiquitylation in which Hsc70 recruits CHIP to Epsin, thereby promoting its ubiquitylation. Indeed, we present both in vitro and in vivo evidence that CHIP promotes Epsin ubiquitylation.

\section{Materials and methods}

Cell lines and reagents. Human embryonic kidney (HEK) 293T cells were cultured as described previously [10]. The following antibodies and reagents were used: mouse monoclonal anti-hemagglutinin (HA), anti-Myc antibodies, and mouse anti-ubiquitin antibodies (CovanceBabco, Berkeley, CA); and anti-glutathione- $S$-transferase (GST) antibodies conjugated with horseradish peroxidase (HRP) (Santa Cruz Biotechnology, Santa Cruz, CA), rabbit anti-CHIP antibodies were generously provided by Dr. Cam Patterson (University of North Carolina, Chapel Hill, NC). Purified ubiquitin, N-ethylenediamine (NEM), and protease inhibitors were purchased from Sigma Chemical (St. Louis, MO). DNA gel extraction kits were purchased from Qiagen (Valencia, CA). GSH-agarose was purchased from Amersham Biosciences (Piscataway, NJ). GST antibodies were used as previously described [9].

Constructs. The Myc tagged ubiquitin expression construct, pCW7, was kindly provided by Dr. Ron Kopito and previously described [16]. The expression plasmid encoding HA-tagged Xenopus Epsin was generated as described [17]. Plasmids for bacterial and mammalian expression of CHIP were generously provided by Dr. Cam Patterson (University of North Carolina, Chapel Hill, NC). The expression construct encoding Myc-tagged Drosophila Epsin (pCMV5-2.1.1) was kindly provided by Dr. Jane McGlade. GST-fusion constructs encoding amino acids 1-305 of Epsin (GST-4305), and 1-167 (GSTENTH) and GST alone in pGEX-2TK were used for bacterial expression. GST-Epsin fusion constructs for expression in mammalian cells were previously described [10]. UIM constructs used in experiments are described elsewhere [9]. To generate HA-tagged Hsc70 truncation mutants, the following procedure was employed. pGEXHsc70(1-540), pGEX-Hsc70(373-540), pGEX-Hsc70(540-650), and pGEX-Hsc70(1-373-650) (provided by Ernst Ungewickell, Washington University, St. Louis, MO) were digested with BamHI and NotI. The excised fragments were gel purified and ligated into pCGN-PTB [18] digested BamHI and NotI. However, owing to a frame shift resulting in the loss of an in-frame stop codon for pCGN-Hsc70(1540 ) and pCGN-Hsc70(373-540), a 74 amino acid extension is present at the carboxy-terminus for these proteins. To reduce this 74 amino acid extension, these plasmids were digested with SalI and NotI, bluntended with Klenow, and then re-ligated with T4 ligase (New England Biolabs). This yields HA-tagged Hsc70(1-540) and Hsc70(373-540) proteins with a 9 amino acid addition at the $\mathrm{C}$-terminal end. These constructs were confirmed by DNA sequence analysis.

Cell culture. HEK 293T cells were plated at a density of $2 \times 10^{6}$ cells per $100 \mathrm{~mm}$ cell culture dish and transiently transfected by the calcium phosphate precipitation method [18] with the indicated expression constructs. Forty-eight hours post-transfection, cells were washed with warm PBS and lysed in cold PLC-LB (50 mM Hepes, pH 7.5, $150 \mathrm{mM}$ $\mathrm{NaCl}, 10 \%$ glycerol, $1 \%$ Triton X-100, 1 mM EGTA, $1.5 \mathrm{mM} \mathrm{MgCl}$, and $100 \mathrm{mM} \mathrm{NaF}$ ) supplemented with $1 \mathrm{mM}$ sodium orthovanadate, $10 \mu \mathrm{g} / \mathrm{ml}$ leupeptin, $10 \mu \mathrm{g} / \mathrm{ml}$ aprotinin, $1 \mathrm{mM}$ PMSF, $1 \mathrm{mM}$ benzamidine, and $5 \mathrm{mM}$ NEM. After $20 \mathrm{~min}$ at $4{ }^{\circ} \mathrm{C}$, insoluble debris was removed by centrifugation, and lysates were stored at $-80^{\circ} \mathrm{C}$ until analysis. Lysate protein concentrations were determined using the Pierce protein assay kit (Pierce, Rockford, IL).

Immunoprecipitation and immunoblotting. For immunoprecipitation analysis, equal amounts of lysate protein $(1 \mathrm{mg})$ were pre-cleared with protein-A or -G-agarose (Sigma) for $1 \mathrm{~h}$ at $4{ }^{\circ} \mathrm{C}$. Pre-cleared lysates were then incubated with the appropriate antibody for $1 \mathrm{~h}$ at $4{ }^{\circ} \mathrm{C}$. Protein-A or -G-agarose was added to immune complexes and incubated an additional $1 \mathrm{~h}$ at $4{ }^{\circ} \mathrm{C}$, and then washed five times with PLCLB supplemented with protease inhibitors and NEM. Under certain conditions, immunoprecipitations and washes were performed in PLC-LB (with inhibitors) supplemented with $1 \%$ sodium deoxycholate and $0.1 \%$ SDS (final concentrations). Immunoprecipitated proteins were heated at $70^{\circ} \mathrm{C}$ for $10 \mathrm{~min}$ in LDS sample buffer (Invitrogen, Carlsbad, CA) supplemented with 5\% $\beta$-mercaptoethanol (v/v). Protein samples were resolved by SDS-PAGE and transferred onto Immobilon-P PVDF membranes (Millipore, Bedford, MA). After blocking membranes in TBST $(20 \mathrm{mM}$ Tris, $\mathrm{pH} 7.5,0.9 \% \mathrm{NaCl}$, and $0.1 \%$ Tween 20 ) containing $3 \%$ non-fat milk powder, immunoblotting was performed with the appropriate primary antibody in TBST containing 3\% non-fat milk powder. Membranes were washed and incubated with the appropriate secondary antibody conjugated with HRP in TBST. Following extensive washing, an enhanced chemiluminescence system (SuperSignal, Pierce) was used for protein detection. 
In vitro ubiquitylation assay. Ubiquitylation assays were carried out as described [19], with some modifications. Bacterially expressed His-tagged CHIP $(4 \mu \mathrm{M})$ was incubated with $2 \mu \mathrm{M}$ bacterially expressed GST-Epsin $\triangle 305$, GST-ENTH, or GST alone in the presence of $0.1 \mu \mathrm{M}$ purified rabbit E1 (Calbiochem, La Jolla, CA), $8 \mu \mathrm{M}$ purified E2 enzyme UbcH5a (Boston Biochem, Cambridge, MA) or GST-tagged UbcH5a (Biomol, Plymouth Meeting, PA), $4 \mu \mathrm{M}$ each of Hsc/Hsp70 and Hsp40 (Stressgen, Vancouver, BC), $2.5 \mathrm{mg} / \mathrm{ml}$ ubiquitin (Sigma), and $5 \mathrm{mM}$ ATP in ubiquitylation assay buffer (20 mM Mops, pH 7.2, $100 \mathrm{mM} \mathrm{KCl}, 5 \mathrm{mM} \mathrm{MgCl}_{2}$, and $10 \mathrm{mM}$ dithiothreitol) in $10 \mu \mathrm{l}$ reaction volumes for $4 \mathrm{~h}$ at $30^{\circ} \mathrm{C}$. Ubiquitylated protein was purified by GSH-pulldown, and washed several times in PLC-LB containing protease inhibitors and NEM, followed by the addition of SDS-PAGE sample buffer containing $6 \mathrm{M}$ urea. For reactions using HA-Epsin $\Delta 305$, aliquots were immediately combined with SDS-PAGE sample buffer containing $6 \mathrm{M}$ urea at the end of the reaction period. Samples were heated at $70{ }^{\circ} \mathrm{C}$ and then fractionated by SDS-PAGE. Immunoblotting was performed with either HRP-conjugated anti-GST or anti-HA antibodies where appropriate. To confirm that shifted bands correspond to ubiquitylated Epsin protein, linear regression analysis was performed to calculate molecular weight as a function of the migration distance of the shifted bands.

\section{Results}

\section{Epsin interacts with Hsc70 through its UIMs}

While attempting to map the ubiquitylation sites in Epsin by mass spectrometry, we identified $\mathrm{Hsc} 70$ as an Epsin-associated protein (data not shown). To examine this interaction in more detail, we immunoprecipitated epitope-tagged Epsin from cells and examined the association with endogenous Hsc70 (Fig. 1). Both Myctagged and HA-tagged Epsin isoforms associated with endogenous Hsc70 in cell lysates (Fig. 1). Furthermore, this interaction was dependent on the presence of the UIMs (Fig. 1B). Indeed, chimeric fusion proteins of Epsin's UIMs with yellow fluorescent protein (YFP) specifically associated with Hsc70 whereas the UIMs from other proteins associated with Hsc70 only weakly or not at all (Fig. 1C and data not shown).

To determine which region of $\mathrm{Hsc} 70$ interacted with Epsin, we co-expressed Myc-tagged Epsin and HA-tagged truncation mutants of Hsc70 in 293T cells (Fig. 2). Immunoprecipitation of Myc-Epsin resulted in the co-precipitation of Hsc70 mutants 373-540 and 373-650. However, we did not detect association of Myc-Epsin with Hsc70 540-650, suggesting that the interaction was specifically mediated by amino acids 373-540 which encompasses the substrate-binding domain of Hsc70. Interestingly, we did not detect association of Hsc70 1-540, suggesting that the presence of the $\mathrm{NH}_{2}$-terminal ATPase domain in the absence of the $\mathrm{COOH}$-terminal domain inhibits the interaction of this truncation mutant with Epsin. In the converse experiments, immunoprecipitation of the HA-tagged Hsc70 373-540 and 373-650 mutants co-precipitated Myc-Epsin. Consistent with the prior experiments, the $\mathrm{Hsc70}$
A
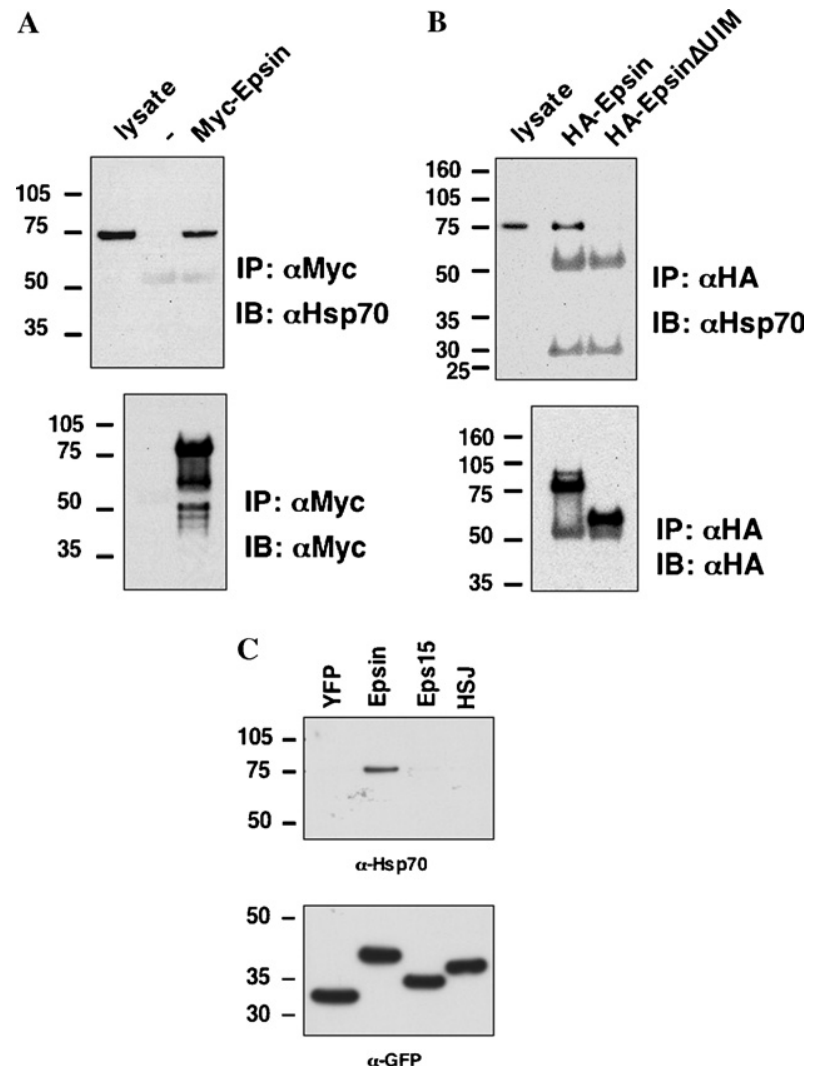

Fig. 1. Epsin association with Hsc70. (A) Cells were transfected with Myc-tagged Drosophila Epsin and harvested 48 h later. Non-transfected cells were used as a negative control. Samples were immunoprecipitated with anti-Myc antibodies and then probed with anti-Hsp70 (top panel) or anti-Myc (bottom panel). For immunoblotting with anti-Hsp70 antibodies, a non-transfected cell lysate was included as a positive control for Hsc70 detection. (B) Similar to (A) except cells were transfected with HA-tagged Xenopus Epsin or a deletion mutant lacking the three UIMs (HA-Epsin$\Delta$ UIM) and harvested $48 \mathrm{hr}$ later. Samples were immunoprecipitated with anti-HA antibodies and then probed with anti-Hsp70 (top panel) or anti-HA (bottom panel). The slower migrating HA-reactive band in the full-length Epsin sample corresponds to monoubiquitylated Epsin. (C) Cells were transfected with YFP-tagged Epsin UIM, Eps15 UIM or HSJ UIM and harvested $48 \mathrm{~h}$ later. Samples were immunoprecipitated with anti-GFP antibodies and then probed with anti-Hsp70 (top panel) or anti-GFP (bottom panel).

1-540 and 540-650 mutants did not associate with Myc-Epsin.

\section{CHIP ubiquitylates Epsin in vitro}

The carboxy-terminus of Hsc70 interacts with the E3 ubiquitin ligase CHIP through its tetratricopeptide repeat (TPR) domains [19]. This interaction is important for CHIP's role in protein quality control [20] and for ubiquitylation of numerous substrates [21-23]. Given the interaction of Hsc70 with Epsin, we tested whether CHIP was capable of ubiquitylating Epsin in vitro. As illustrated in Fig. 3A, CHIP ubiquitylated GST-Epsin in an ATP-dependent manner. In the absence of CHIP, 

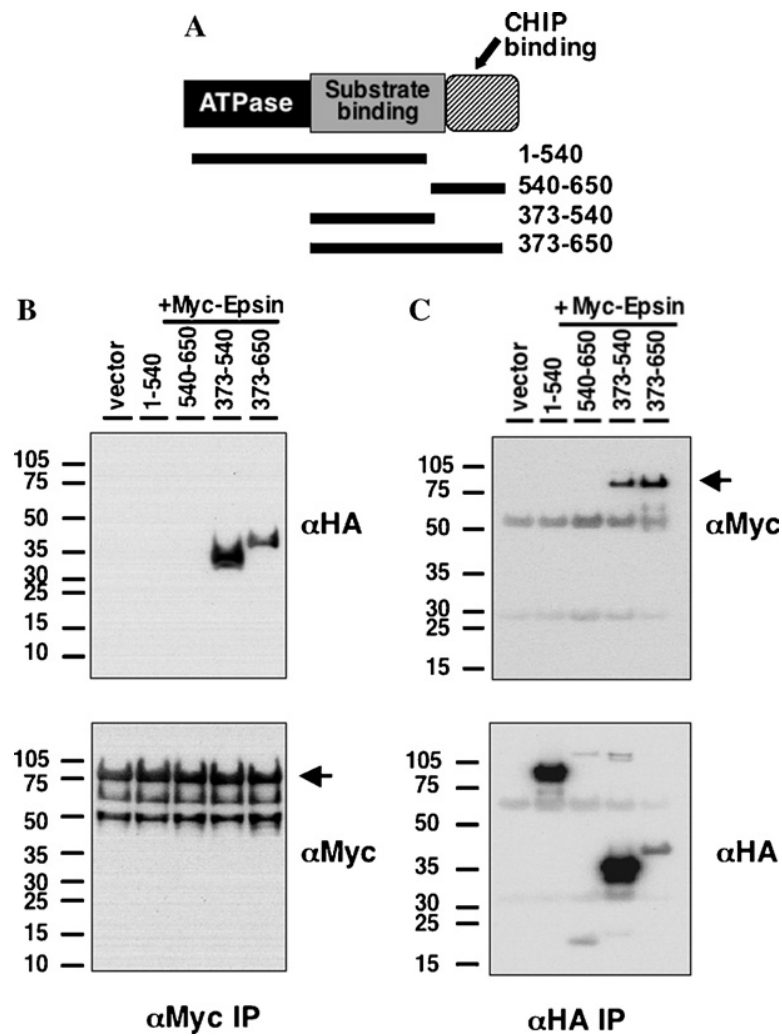

Fig. 2. Epsin interacts with the substrate binding domain of Hsc70. (A) Domain structure of Hsc70. Indicated below are the Hsc70 deletion constructs used to map the site of interaction with Epsin. (B) HEK 293T cells were co-transfected with Myc-tagged Drosophila Epsin together with one of the four HA-epitope-tagged Hsc70 deletion constructs as indicated. Forty-eight hours after transfection, cells were harvested and samples were immunoprecipitated with anti-Myc antibodies. Immunoprecipitates were then probed with anti-HA (top panel) or anti-Myc (bottom panel) to determine the assocation with Hsc70 truncation mutants. (C) The converse experiment of that shown in (B). HA-tagged Hsc70 mutants were immunoprecipitated with antiHA antibodies and Epsin association was determined by Western blot analysis with anti-Myc antibodies (top panel). The level of the Hsc70 truncation mutants was determined by Western blot analysis with HA antibodies (bottom panel). The arrows in (B), (C) mark the positions of Myc-Epsin.

low levels of Epsin monoubiquitylation were detected. This result is consistent with prior observations of E2s capable of ubiquitylating substrates in an E3-independent manner $[24,25]$. However, the addition of CHIP dramatically increased Epsin ubiquitylation. In the presence of GST-tagged UbcH5, CHIP predominantly monoubiquitylated Epsin. However, in the presence of non-tagged UbcH5, CHIP polyubiquitylated Epsin. Similar results were obtained using HA-tagged Epsin $\Delta 305$ as the substrate (Fig. 3B). Although we consistently observed different ubiquitylation patterns for Epsin when using GST-UbcH5 as compared to untagged $\mathrm{UbcH} 5$, we do not believe that this difference is physiologically relevant but is rather due to the fact that the presence of the GST tag may alter the activity of UbcH5 through promoting dimerization of the protein. Addi-

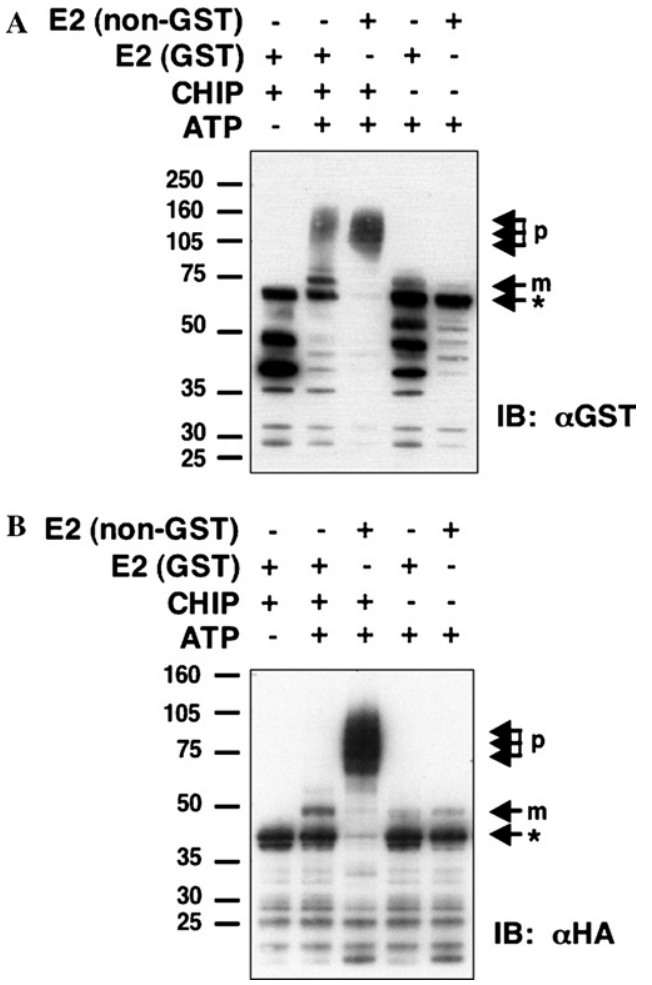

Fig. 3. Epsin is ubiquitylated by CHIP in vitro. A bacterialy expressed GST-fusion (A) or HA-tagged (B) protein of Epsin encompassing the ENTH domain and UIMs (amino acids 1-305; hereafter referred to as GST- $\Delta 305$ or HA- $\Delta 305$ ) was used as a substrate for the in vitro ubiquitylation reactions. Each lane represents an assay sample containing E2 conjugating enzyme (UbcH5; either GST-tagged or untagged), ATP, and CHIP where indicated. E1 enzyme and ubiquitin were present in all samples. For reactions using GST- $\Delta 305$ as the substrate (A), ubiquitylated proteins were purified by GSH-pulldown, washed several times in PLC-LB containing protease inhibitors and NEM, and then resuspended in sample buffer containing urea. For reactions using HA- $\Delta 305$ as substrate (B), sample buffer containing urea was added directly to samples followed by heating at $70^{\circ} \mathrm{C}$ for $15 \mathrm{~min}$. Proteins were then fractionated by SDS-PAGE, transferred to PVDF membranes, and then probed with either anti-GST (A) or antiHA antibodies (B). Mono- and poly-ubiquitylated species of Epsin are indicated in both panels by the $\mathrm{m}$ and $\mathrm{p}$, respectively. Unmodified Epsin is indicated by an asterisk.

tionally, these two forms of $\mathrm{UbcH} 5$ were obtained from different commercial sources (see Materials and methods), and thus may have different specific activities although these activities were not measured directly. Given the importance of Hsc/Hsp70 and Hsp40 in CHIP function, we examined the role of chaperones in CHIPdependent ubiquitylation of Epsin in vitro (Fig. 4). Although Epsin ubiquitylation was dependent on the presence of the UIMs consistent with our prior cell-based experiments [10], chaperones were not required for in vitro ubiquitylation of Epsin by CHIP as revealed by CHIP-mediated polyubiquitylation in the absence of Hsp40 and/or Hsc/Hsp70 (Fig. 4).

In multiple in vitro experiments, CHIP consistently formed polyubiquitylated species of Epsin with a maxi- 


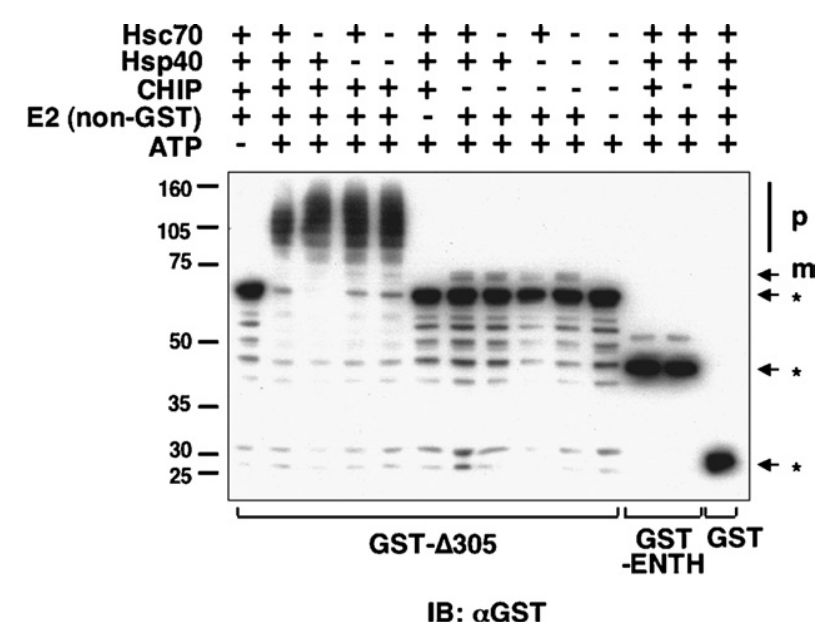

Fig. 4. CHIP-mediated ubiquitylation of Epsin is UIM-dependent but chaperone-independent. Ubiquitylation reactions containing the indicated components were performed as described in Fig. 3. GST fusion

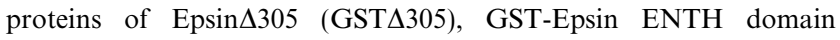
(GST-ENTH), or GST alone were used as substrates in the reactions as indicated. Proteins were fractionated by SDS-PAGE and then transferred to PVDF (Immobilon-P) membranes, followed by immunoblotting with an anti-GST antibody. Unmodified GST proteins are marked by asterisks. Mono- and poly-ubiquitylated GST proteins are indicated by the $\mathrm{m}$ and $\mathrm{p}$, respectively.

mum observed molecular weight of approximately $160 \mathrm{kDa}$ after $4 \mathrm{~h}$ incubation. To account for this apparent limitation to the extent Epsin is ubiquitylated, we performed in vitro time-course experiments to determine what ubiquitylated species of Epsin are formed by CHIP at early and later time points. Both GST-tagged and HA-tagged Epsin $\triangle 305$ (Figs. 5A and B, respectively) were ubiquitylated in vitro reaching a maximum molecular weight of $\sim 160 \mathrm{kDa}$ after $2-4 \mathrm{~h}$. At this time, all of Epsin $\Delta 305$ was ubiquitylated; however, at $30 \mathrm{~min}$ a significant fraction of Epsin $\Delta 305$ was unmodified. Interestingly, Epsin $\Delta 305$ is predominantly monoubiquitylated at $30 \mathrm{~min}$, with some di- and tri-ubiquitylated species formed as well.

\section{CHIP promotes Epsin ubiquitylation in vivo}

Given the in vitro ubiquitylation of Epsin by CHIP, we next tested whether CHIP ubiquitylated Epsin in vivo. In cells co-expressing GST-Epsin and HA-tagged ubiquitin, we detected increased levels of slower migrating forms of Epsin that were each shifted by approximately $10 \mathrm{kDa}$, consistent with covalent attachment of HA-ubiquitin (Fig. 6A). Interestingly, the slower migrating species that are increased with CHIP overexpression correspond to polyubiquitylated GST-Epsin. Western blot analysis of these immunopurified proteins with antibodies to tagged ubiquitin revealed that these shifted bands did indeed correspond to ubiquitylated Epsin (Fig. 6A, middle panel). Overexpression of CHIP in $293 \mathrm{~T}$ cells led to a dose-dependent increase in ubiqui-
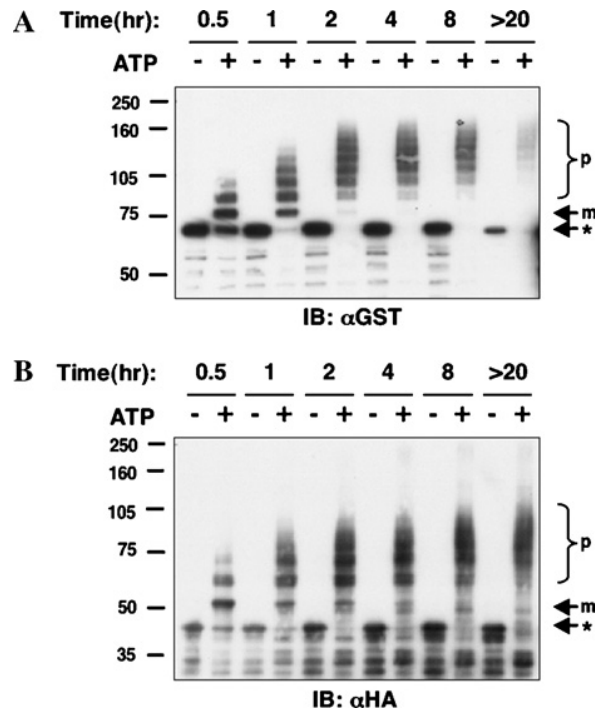

Fig. 5. Time-dependent Epsin ubiquitylation by CHIP in vitro. Ubiquitylation reactions containing the indicated components were performed as described in Fig. 3. A GST fusion protein of Epsin $\Delta 305$ (GST $\Delta 305)$ (A) or HA-tagged Epsin $\Delta 305$ (HA- $\Delta 305$ ) (B) were used as substrates in the reactions, and the reactions were allowed to proceed for the indicated time periods. Proteins were fractionated by SDSPAGE and then transferred to PVDF (Immobilon-P) membranes, followed by probing for either GST- $\Delta 305$ (A) or HA- $\Delta 305$ (B) using the appropriate antibody. Unmodified $E p \sin \Delta 305$ protein is marked by asterisks. Mono- and poly-ubiquitylated Epsin $\Delta 305$ proteins are indicated by the $\mathrm{m}$ and $\mathrm{p}$, respectively.

tylation of GST-Epsin; however, free GST was not modified by CHIP as revealed by in vitro experiments (Fig. 4). The modification of GST-Epsin occurred on the Epsin portion of the fusion protein as revealed by thrombin cleavage of GST-Epsin (Fig. 6B). We also tested Epsin ubiquitylation by CHIP using YFP-tagged Epsin and Myc-tagged ubiquitin, obtaining similar results. In cells overexpressing CHIP, slower migrating forms of Epsin shifted by more than $10 \mathrm{kDa}$ were observed (Fig. 7), confirming the results with GST-Epsin and HA-tagged ubiquitin. Lastly, deletion of the UIMs resulted in a dramatic reduction in ubiquitylated Epsin, and CHIP overexpression had no effect on this low level of ubiquitylated Epsin (Fig. 7).

\section{Discussion}

The E3 ubiquitin ligase CHIP was first identified as a TPR-containing protein found to inhibit the ATPase, substrate binding, and protein refolding activities of Hsc/Hsp70 [26], indicating that CHIP plays an important function in chaperone-dependent protein quality control [20,21]. CHIP E3 ubiquitin ligase activity requires its carboxy-terminal U-box domain, functions in collaboration with the $\mathrm{UbcH} 5$ family of E2 ubiquitin-conjugating enzymes, and directly ubiquitylates 
A
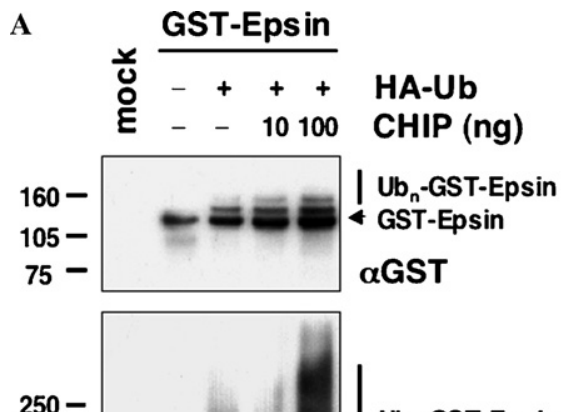

B

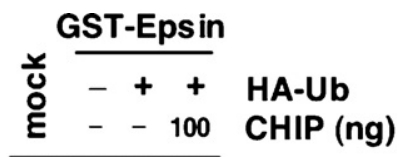

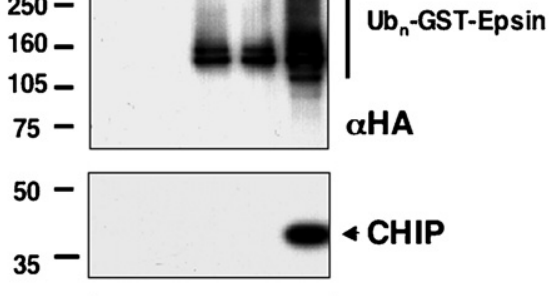

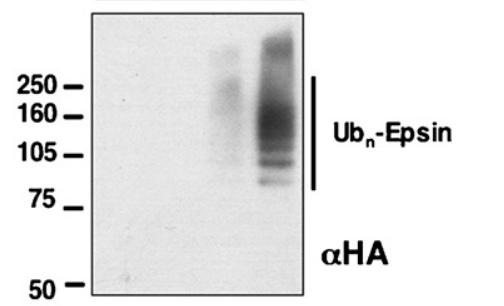

Fig. 6. CHIP overexpression increased levels of ubiquitylated Epsin. (A) HEK 293T cells were co-transfected with GST-tagged Epsin, HA-tagged ubiquitin (HA-Ub), and increasing concentrations of Myc-tagged CHIP. Forty-eight hours after transfection, cells were harvested and GST-Epsin was purified with GSH-agarose beads. Precipitated proteins were then eluted off beads with SDS-PAGE sample buffer, fractionated by SDS-PAGE, and transferred to PVDF (Immobilon-P) membranes. Immunoblotting was performed using anti-GST (top panel), anti-HA (second panel), and antiMyc (third panel) antibodies to detect GST-Epsin, HA-ubiquitin, and Myc-CHIP, respectively. The slower migrating species marked with arrows in the top panel react with the HA antibodies in the second panel. HA-ubiquitin was expressed at equal levels in all samples (bottom panel) (B) GST-Epsin was purified from cells following co-expression with various levels of CHIP. A fraction of the total precipitated protein was resuspended in SDS-PAGE sample buffer and analyzed for the covalent attachment of ubiquitin by Western blot analysis with anti-HA antibodies (top panel). The remaining GST-Epsin was subjected to thrombin cleavage as previously described [10] to release Epsin from GST. The soluble portion of the reaction containing Epsin was fractionated on SDS-PAGE, transferred to membranes, and then probed with anti-HA antibodies (bottom panel) to detect ubiquitylated Epsin.
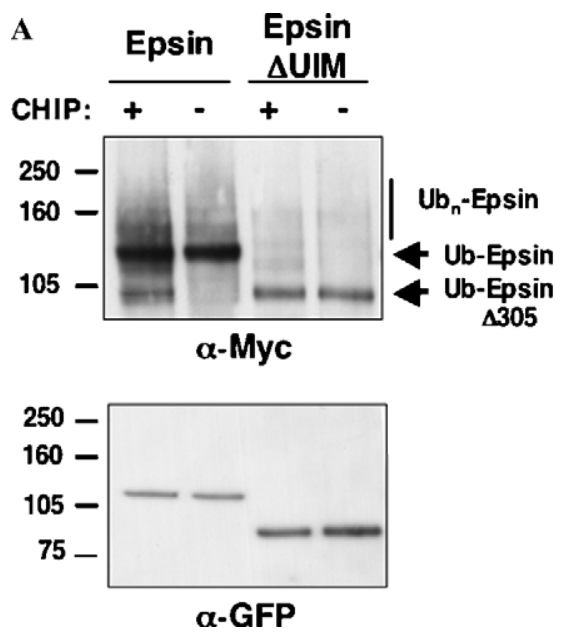
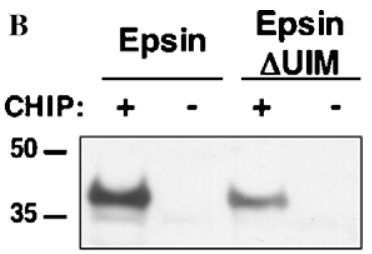

$\alpha-$ CHIP

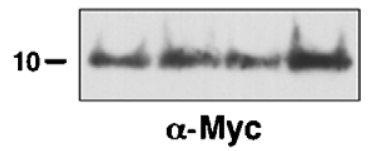

Fig. 7. CHIP increases ubiquitylation of Epsin. Analysis was performed as described in Fig. 5. Cells were co-transfected with YFP-tagged Epsin or Epsin $\Delta$ UIM, Myc-tagged ubiquitin (Myc-Ub), and Myc-tagged CHIP. Forty-eight hours after transfection, cells were harvested in lysis buffer. (A) Immunopurified YFP-Epsin or Epsin $\Delta$ UIM was then analyzed for the attachment of Myc-Ub. Western blot analysis was performed using anti-Myc (top panel) to detect Ub or anti-GFP (bottom panel) for total levels of Epsin. To minimize co-precipitation of associated ubiquitylated proteins, immunoprecipitations were performed in buffer supplemented with $1 \%$ sodium deoxycholate and $0.1 \%$ SDS (final concentrations). (B) Total cell lysates were examined for the level of expression of Myc-CHIP ( $\alpha$-CHIP) and Myc-Ub ( $\alpha$-Myc).

Hsc/Hsp70 without affecting protein half-life [19,21]. In addition to its function in protein quality control, CHIP regulates proteins involved in cell signaling $[22,23,27-$ 31], cellular architecture [32], and chaperone-mediated physiology [26,33-35]. Our finding that CHIP ubiquitylates Epsin indicates that CHIP has functions beyond protein quality control that may include the regulation of adaptor proteins involved in endocytosis. 
Several groups have investigated the involvement of Hsc70 in endocytosis and have uncovered a role for this chaperone in the uncoating of clathrin triskelia from clathrin-encased intracellular transport vesicles [36-39]. An important step for this uncoating process is the binding of auxilin to assembled clathrin, initiating Hsc70-dependent release of the triskelia from the vesicle membrane [40]. This latter process involves stimulation of Hsc70 ATPase activity upon binding with auxilin [41], and the interaction occurs between the $\mathbf{J}$ domain of auxilin and the ATPase and substrate-binding domains of Hsc70 [42]. Further evidence of the role for Hsc70 in clathrin uncoating comes from studies showing that cytosolic depletion of Hsc70 inhibits clathrin uncoating [43], the identification of dominant interfering Hsc70 mutants [44], and genetic data supporting the role for Hsc70 in endocytosis and clathrin uncoating [45]. In view that CHIP ubiquitylates Hsc70 [19], CHIP might therefore assist in the regulation of endocytosis. Our observation of Hsc70 interacting with the UIMs of Epsin suggests a mechanism by which CHIP is recruited to epsin. The loss of CHIP-dependent Epsin ubiquitylation by deletion of Epsin's UIMs (Fig. 7) supports this idea; however, we were unable to isolate a complex comprised of all three proteins. As the UIM has weak affinity for ubiquitin [46], we believe that the lack of detection of this complex is due to low affinity, transient interactions. Furthermore, Epsin is necessary for membrane curvature and clathrin-coated pit formation [47], recruits activated receptors to clathrin-coated pits [3], and facilitates recruitment of other endocytic adaptors during the maturation stages of clathrin-coated vesicle formation (reviewed in [5]). Our observation that Epsin is ubiquitylated by CHIP provides additional support for a role for CHIP in clathrin-mediated endocytosis. However, further experimentation is necessary to explore this possibility.

The interaction between Hsc70 and Epsin in vivo was found to depend on Epsin's UIMs, consistent with the observation that only the UIMs of Epsin specifically bind Hsc70 (Fig. 1). Given that Epsin's UIMs bind ubiquitin in vitro and that this activity is necessary for Epsin ubiquitylation [9], these two observations suggest Epsin ubiquitylation depends on both Hsc70 and ubiquitinbinding by the UIMs. The domain of Hsc70 that binds Epsin was mapped to the substrate-binding region (Fig. 2), providing further evidence of the specificity of the interaction between the two proteins. Interestingly, a protein construct consisting of both the ATPase domain and the substrate-binding region but lacking the CHIP-binding domain did not immunoprecipitate with Epsin, suggesting that the ATPase domain is inhibitory to the interaction. It is unknown whether this $\mathrm{Hsc} 70 \mathrm{mu}-$ tant has altered function or conformation relating to its ability to acquire a high substrate affinity (ADP-bound) form from a low affinity (ATP-bound) state, in vivo.
However, in vitro this mutant exhibits decreased affinity for known Hsc70 substrates [42]. Nonetheless, endogenous (full-length) Hsc70 interacted with Epsin in a UIM-dependent manner, and the interaction correlates with full-length Epsin being monoubiquitylated in cells as evidenced by the single shifted Epsin band (Fig. 1B, lower panel, lane 1). These data support our hypothesis that the interaction between Epsin and Hsc70 recruits CHIP to Epsin, leading to its ubiquitylation. The lack of a requirement for Hsc70 for Epsin ubiquitylation in vitro would contradict this idea. However, it is important to keep in mind that all components for in vitro ubiquitylation by CHIP are in molar excess relative to Epsin, hence precluding the need for $\mathrm{Hsc} 70$ to recruit CHIP to Epsin in order for it to be ubiquitylated by CHIP. The lack of CHIP-mediated polyubiquitylation of Epsin's ENTH domain alone or GST (Fig. 4, lanes 12 and 14, respectively) demonstrates a degree of specificity for CHIP-mediated Epsin ubiquitylation in vitro, in that the UIMs are necessary for modification of Epsin by CHIP.

We did not observe changes in the levels of YFP- or GST-tagged Epsin with CHIP overexpression nor did we observe any effect of proteasome inhibition on levels of either Epsin or ubiquitylated Epsin consistent with the finding that CHIP-mediated ubiquitylation of Hsc70 does not result in its degradation or targeting to the proteasome [19]. Taken together, these data indicate that CHIP ubiquitylation of Epsin, like that of Hsc70, does not target Epsin to the proteasome. These results are consistent with previous reports by ourselves and others demonstrating that Epsin ubiquitylation does not lead to its degradation $[10,11]$. Discordance exists, however, between these reports demonstrating Epsin monoubiquitylation and the data presented here showing increased Epsin polyubiquitylation by CHIP. While this discrepancy might exclude CHIP as an E3 ligase for Epsin, genetic data in Drosophila support the notion that the formation of monoubiquitylated Epsin requires deubiquitylating enzymes (DUBs), implying that Epsin is first polyubiquitylated and then processed to the mono-ubiquitylated form by one or more DUBs (Fig. 8). Indeed, recent work from our group has identified a DUB which can process polyubiquitylated Epsin to smaller mono- and di-ubiquitylated species in vitro (unpublished observation). Furthermore, in vitro timecourse data indicate that CHIP polyubiquitylates Epsin by extending ubiquitin chains (Fig. 5), akin to an "E4" ubiquitin ligase activity seen with the yeast protein UFD2 [48]. Hence, CHIP fits the criteria as an E3 ubiquitin ligase functioning in the first step of this model. Accordingly, CHIP overexpression would favor increased Epsin ubiquitylation, which we observed in this study (Figs. 6 and 7).

Efforts have been made to address the mechanism(s) of Epsin deubiquitylation and to identify DUBs in- 


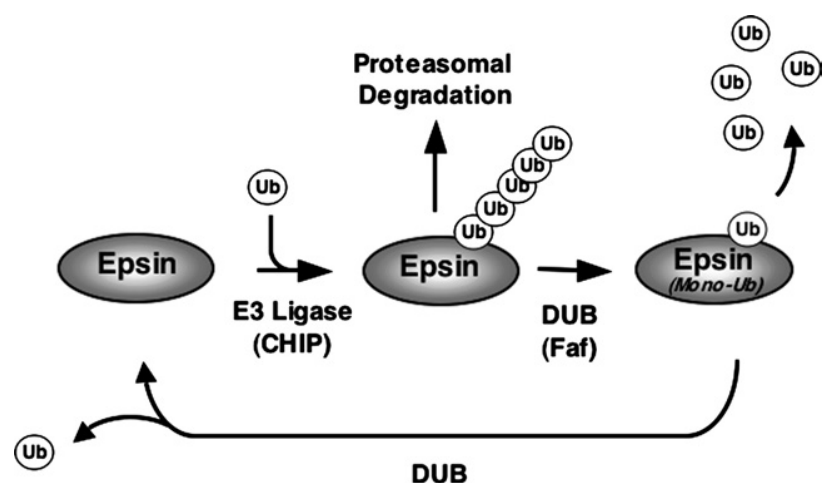

Fig. 8. Model for Epsin ubiquitylation by E3 ubiquitin ligases such as CHIP. Epsin is ubiquitylated by an E3 ubiquitin ligase such as CHIP, resulting in the addition of a polyubiquitin chain. While this may target Epsin for degradation by the proteasome, genetic data in Drosophila suggest that a de-ubiquitylating enzyme (DUB) such as Fat facets (Faf) may function to shorten the polyubiquitin chain to produce monoubiquitylated Epsin. This cleavage process may proceed to completion, recycling Epsin back to its unmodified form.

volved in this process. Currently, Fam, the mouse homologue of the Drosophila DUB Fat facets (Faf), is the most-likely DUB mediating Epsin deubiquitylation. It was recently shown that silencing Fam by siRNA abrogated ionomycin-induced decrease in Epsin ubiquitylation, indicating that Fam plays a role in regulating levels of Epsin ubiquitylation [15]. Moreover, the Faf gene can be substituted with a transgene encoding Fam [49], lending further support to the role of this protein in Epsin deubiquitylation. However, in vitro data on direct deubiquitylation of Epsin by Fam are lacking, and the $\sim 50 \%$ identity between Faf and Fam suggests different specificities for substrates [49]; indeed, the only reported substrates for Fam are AF-2 and $\beta$-catenin $[50$ 52]. Nonetheless, structure-function studies of Faf suggest that it may either bind directly to Lqf or bind to a protein complex containing Lqf $[7,53]$. In the latter case, it is conceivable that such a complex might include the ubiquitin ligase(s) responsible for Lqf ubiquitylation. Following on this idea, a protein complex consisting of both E3 ubiquitin ligase(s) and DUBs would serve as a convenient mechanism to regulate the extent of substrate ubiquitylation by an E3 ubiquitin ligase. This would be consistent with observations of Faf antagonizing both ubiquitylation and proteolysis [54,55], and recent reports of DUBs directly interacting with E3 ubiquitin ligases [56,57].

The abundance of E3 ligases in eukaryotes raises the possibility that Epsin may be ubiquitylated by additional E3 ligases. Indeed, others have reported that Epsin is ubiquitylated by the HECT domain E3 ligase NEDD4 [11]. However, our data indicate that CHIP is also competent for ubiquitylating Epsin. This activity is UIM-dependent, corroborating our previous findings for the importance of the UIMs in Epsin ubiquitylation [10]. Further investigation of the cellular consequences of CHIP-mediated ubiquitylation of Epsin, which at present are unknown, as well as studies investigating the function of ubiquitylated Epsin, will provide important insights into the function of ubiquitylation in endocytosis.

\section{Acknowledgments}

The authors thank Drs. Cam Patterson and Ernst Ungewickell for providing reagents. We also thank Dr. Cam Patterson for assistance with the in vitro ubiquitylation assays; Drs. Ken Tomer and Leesa Deterding for the mass spec analysis; Drs. David Armstrong and Trevor Archer for comments on the manuscript; and members of the O'Bryan lab for helpful discussions and suggestions.

\section{References}

[1] F.M. Brodsky, C.Y. Chen, C. Knuehl, M.C. Towler, D.E. Wakeham, Biological basket weaving: formation and function of clathrin-coated vesicles, Annu. Rev. Cell Dev. Biol. 17 (2001) 517568.

[2] S.L. Schmid, Clathrin-coated vesicle formation and protein sorting: an integrated process, Annu. Rev. Biochem. 66 (1997) 511-548.

[3] H. Chen, S. Fre, V.I. Slepnev, M.R. Capua, K. Takei, M.H. Butler, P.P. Di Fiore, P. De Camilli, Epsin is an EH-domainbinding protein implicated in clathrin-mediated endocytosis, Nature London 394 (1998) 793-797.

[4] T. Itoh, S. Koshiba, T. Kigawa, A. Kikuchi, S. Yokoyama, T. Takenawa, Role of the ENTH domain in phosphatidylinositol4,5-bisphosphate binding and endocytosis, Science 291 (2001) $1047-1051$.

[5] V.I. Slepnev, P. De Camilli, Accessory factors in clathrindependent synaptic vesicle endocytosis, Nat. Rev. Neurosci. 1 (2000) 161-172.

[6] L. Hicke, R. Dunn, Regulation of membrane protein transport by ubiquitin and ubiquitin-binding proteins, Annu. Rev. Cell Dev. Biol. 19 (2003) 141-172.

[7] X. Chen, B. Zhang, J.A. Fischer, A specific protein substrate for a deubiquitinating enzyme: liquid facets is the substrate of Fat facets, Genes Dev. 16 (2002) 289-294.

[8] A.L. Cadavid, A. Ginzel, J.A. Fischer, The function of the Drosophila fat facets deubiquitinating enzyme in limiting photoreceptor cell number is intimately associated with endocytosis, Development 127 (2000) 1727-1736.

[9] S.L. Miller, E. Malotky, J.P. O'Bryan, Analysis of the role of ubiquitin-interacting motifs in ubiquitin binding and ubiquitylation, J. Biol. Chem. 279 (2004) 33528-33537.

[10] C.E. Oldham, R.P. Mohney, S.L. Miller, R.N. Hanes, J.P. O'Bryan, The ubiquitin-interacting motifs target the endocytic adaptor protein epsin for ubiquitination, Curr. Biol. 12 (2002) $1112-1116$.

[11] S. Polo, S. Sigismund, M. Faretta, M. Guidi, M.R. Capua, G. Bossi, H. Chen, P. De Camilli, P.P. Di Fiore, A single motif responsible for ubiquitin recognition and monoubiquitination in endocytic proteins, Nature London 416 (2002) 451-455.

[12] S.C. Shih, K.E. Sloper-Mould, L. Hicke, Monoubiquitin carries a novel internalization signal that is appended to activated receptors, EMBO J. 19 (2000) 187-198. 
[13] S.C. Shih, D.J. Katzmann, J.D. Schnell, M. Sutanto, S.D. Emr, L. Hicke, Epsins and Vps27p/Hrs contain ubiquitin-binding domains that function in receptor endocytosis, Nat. Cell Biol. 4 (2002) 389-393.

[14] J. Terrell, S. Shih, R. Dunn, L. Hicke, A function for monoubiquitination in the internalization of a $G$ protein-coupled receptor, Mol. Cell 1 (1998) 193-202.

[15] H. Chen, S. Polo, P.P. Di Fiore, P.V. De Camilli, Rapid $\mathrm{Ca}^{2+}$ dependent decrease of protein ubiquitination at synapses, Proc. Natl. Acad. Sci. USA 100 (2003) 14908-14913.

[16] C.L. Ward, S. Omura, R.R. Kopito, Degradation of CFTR by the ubiquitin-proteasome pathway, Cell 83 (1995) 121-127.

[17] A. Adams, J.M. Thorn, M. Yamabhai, B.K. Kay, J.P. O’Bryan, Intersectin, an adaptor protein involved in clathrin-mediated endocytosis, activates mitogenic signaling pathways, J. Biol. Chem. 275 (2000) 27414-27420.

[18] J.P. O'Bryan, Q.T. Lambert, C.J. Der, The Src homology 2 and phosphotyrosine binding domains of the ShcC adaptor protein function as inhibitors of mitogenic signaling by the epidermal growth factor receptor, J. Biol. Chem. 273 (1998) 20431-20437.

[19] J. Jiang, C.A. Ballinger, Y. Wu, Q. Dai, D.M. Cyr, J. Hohfeld, C. Patterson, CHIP is a U-box-dependent E3 ubiquitin ligase: identification of Hsc70 as a target for ubiquitylation, J. Biol. Chem. 276 (2001) 42938-42944.

[20] D.M. Cyr, J. Hohfeld, C. Patterson, Protein quality control: Ubox-containing E3 ubiquitin ligases join the fold, Trends Biochem. Sci. 27 (2002) 368-375.

[21] S. Murata, Y. Minami, M. Minami, T. Chiba, K. Tanaka, CHIP is a chaperone-dependent E3 ligase that ubiquitylates unfolded protein, EMBO Rep. 2 (2001) 1133-1138.

[22] P. Connell, C.A. Ballinger, J. Jiang, Y. Wu, L.J. Thompson, J. Hohfeld, C. Patterson, The co-chaperone CHIP regulates protein triage decisions mediated by heat-shock proteins, Nat. Cell Biol. 3 (2001) 93-96.

[23] G.C. Meacham, C. Patterson, W. Zhang, J.M. Younger, D.M. Cyr, The Hsc70 co-chaperone CHIP targets immature CFTR for proteasomal degradation, Nat. Cell Biol. 3 (2001) 100-105.

[24] Z.J. Chen, E.G. Niles, C.M. Pickart, Isolation of a cDNA encoding a mammalian multiubiquitinating enzyme (E225K) and overexpression of the functional enzyme in Escherichia coli, J. Biol. Chem. 266 (1991) 15698-15704.

[25] Z. Chen, C.M. Pickart, A 25-kDa ubiquitin carrier protein (E2) catalyzes multi-ubiquitin chain synthesis via lysine 48 of ubiquitin, J. Biol. Chem. 265 (1990) 21835-21842.

[26] C.A. Ballinger, P. Connell, Y. Wu, Z. Hu, L.J. Thompson, L.Y. Yin, C. Patterson, Identification of CHIP, a novel tetratricopeptide repeat-containing protein that interacts with heat shock proteins and negatively regulates chaperone functions, Mol. Cell. Biol. 19 (1999) 4535-4545.

[27] R. Takahashi, Y. Imai, Pael receptor, endoplasmic reticulum stress, and Parkinson's disease, J. Neurol. 250 (Suppl. 3) (2003) 25-29.

[28] W.K. Sumanasekera, E.S. Tien, J.W. Davis II, R. Turpey, G.H. Perdew, J.P. Vanden Heuvel, Heat shock protein-90 (Hsp90) acts as a repressor of peroxisome proliferator-activated receptor-alpha (PPARalpha) and PPARbeta activity, Biochemistry 42 (2003) 10726-10735.

[29] W. Xu, M. Marcu, X. Yuan, E. Mimnaugh, C. Patterson, L. Neckers, Chaperone-dependent E3 ubiquitin ligase CHIP mediates a degradative pathway for c-ErbB2/Neu, Proc. Natl. Acad. Sci. USA 99 (2002) 12847-12852.

[30] M.J. Lees, D.J. Peet, M.L. Whitelaw, Defining the role for XAP2 in stabilization of the dioxin receptor, J. Biol. Chem. 278 (2003) 35878-35888.

[31] C.P. Cardozo, C. Michaud, M.C. Ost, A.E. Fliss, E. Yang, C. Patterson, S.J. Hall, A.J. Caplan, C-terminal Hsp-interacting protein slows androgen receptor synthesis and reduces its rate of degradation, Arch. Biochem. Biophys. 410 (2003) 134-140.
[32] H. Shimura, D. Schwartz, S.P. Gygi, K.S. Kosik, CHIP-Hsc70 complex ubiquitinates phosphorylated tau and enhances cell survival, J. Biol. Chem. 279 (2004) 4869-4876.

[33] Q. Dai, C. Zhang, Y. Wu, H. McDonough, R.A. Whaley, V. Godfrey, H.H. Li, N. Madamanchi, W. Xu, L. Neckers, D. Cyr, C. Patterson, CHIP activates HSF1 and confers protection against apoptosis and cellular stress, EMBO J. 22 (2003) 54465458.

[34] J. Demand, S. Alberti, C. Patterson, J. Hohfeld, Cooperation of a ubiquitin domain protein and an E3 ubiquitin ligase during chaperone/proteasome coupling, Curr. Biol. 11 (2001) 1569-1577.

[35] H.H. Kampinga, B. Kanon, F.A. Salomons, A.E. Kabakov, C. Patterson, Overexpression of the cochaperone CHIP enhances Hsp70-dependent folding activity in mammalian cells, Mol. Cell. Biol. 23 (2003) 4948-4958.

[36] S. Honing, G. Kreimer, H. Robenek, B.M. Jockusch, Receptormediated endocytosis is sensitive to antibodies against the uncoating ATPase (hsc70), J. Cell Sci. 107 (Pt. 5) (1994) 11851196.

[37] T.G. Chappell, B.B. Konforti, S.L. Schmid, J.E. Rothman, The ATpase core of a clathrin uncoating protein, J. Biol. Chem. 262 (1987) 746-751.

[38] M.Y. Tsai, C. Wang, Uncoupling of peptide-stimulated ATPase and clathrin-uncoating activity in deletion mutant of hsc70, J. Biol. Chem. 269 (1994) 5958-5962.

[39] E. Ungewickell, The 70-kDa mammalian heat shock proteins are structurally and functionally related to the uncoating protein that releases clathrin triskelia from coated vesicles, EMBO J. 4 (1985) 3385-3391.

[40] E. Ungewickell, H. Ungewickell, S.E. Holstein, R. Lindner, K. Prasad, W. Barouch, B. Martin, L.E. Greene, E. Eisenberg, Role of auxilin in uncoating clathrin-coated vesicles, Nature London 378 (1995) 632-635.

[41] R.F. Jiang, T. Greener, W. Barouch, L. Greene, E. Eisenberg, Interaction of auxilin with the molecular chaperone, Hsc70, J. Biol. Chem. 272 (1997) 6141-6145.

[42] E. Ungewickell, H. Ungewickell, S.E. Holstein, Functional interaction of the auxilin $\mathrm{J}$ domain with the nucleotide- and substrate-binding modules of Hsc70, J. Biol. Chem. 272 (1997) 19594-19600

[43] L.A. Hannan, S.L. Newmyer, S.L. Schmid, ATP- and cytosoldependent release of adaptor proteins from clathrin-coated vesicles: a dual role for Hsc70, Mol. Biol. Cell 9 (1998) 2217-2229.

[44] S.L. Newmyer, S.L. Schmid, Dominant-interfering Hsc70 mutants disrupt multiple stages of the clathrin-coated vesicle cycle in vivo, J. Cell Biol. 152 (2001) 607-620.

[45] H.C. Chang, S.L. Newmyer, M.J. Hull, M. Ebersold, S.L. Schmid, I. Mellman, Hsc70 is required for endocytosis and clathrin function in Drosophila, J. Cell Biol. 159 (2002) 477-487.

[46] R.D. Fisher, B. Wang, S.L. Alam, D.S. Higginson, H. Robinson, W.I. Sundquist, C.P. Hill, Structure and ubiquitin binding of the ubiquitin interacting motif, J. Biol. Chem. (2003).

[47] M.G. Ford, I.G. Mills, B.J. Peter, Y. Vallis, G.J. Praefcke, P.R. Evans, H.T. McMahon, Curvature of clathrin-coated pits driven by epsin, Nature London 419 (2002) 361-366.

[48] M. Koegl, T. Hoppe, S. Schlenker, H.D. Ulrich, T.U. Mayer, S. Jentsch, A novel ubiquitination factor, E4, is involved in multiubiquitin chain assembly, Cell 96 (1999) 635-644.

[49] X. Chen, E. Overstreet, S.A. Wood, J.A. Fischer, On the conservation of function of the Drosophila fat facets deubiquitinating enzyme and Fam, its mouse homolog, Dev. Genes Evol. 210 (2000) 603-610.

[50] S. Taya, T. Yamamoto, M. Kanai-Azuma, S.A. Wood, K. Kaibuchi, The deubiquitinating enzyme Fam interacts with and stabilizes beta-catenin, Genes Cells 4 (1999) 757-767.

[51] M. Kanai-Azuma, J.S. Mattick, K. Kaibuchi, S.A. Wood, Colocalization of FAM and AF-6, the mammalian homologues of 
Drosophila faf and canoe, in mouse eye development, Mech. Dev. 91 (2000) 383-386.

[52] S. Taya, T. Yamamoto, K. Kano, Y. Kawano, A. Iwamatsu, T. Tsuchiya, K. Tanaka, M. Kanai-Azuma, S.A. Wood, J.S. Mattick, K. Kaibuchi, The Ras target AF-6 is a substrate of the fam deubiquitinating enzyme, J. Cell Biol. 142 (1998) 1053-1062.

[53] X. Chen, J.A. Fischer, In vivo structure/function analysis of the Drosophila fat facets deubiquitinating enzyme gene, Genetics 156 (2000) 1829-1836.

[54] Z. Wu, Q. Li, M.E. Fortini, J.A. Fischer, Genetic analysis of the role of the Drosophila fat facets gene in the ubiquitin pathway, Dev. Genet. 25 (1999) 312-320.
[55] Y. Huang, R.T. Baker, J.A. Fischer-Vize, Control of cell fate by a deubiquitinating enzyme encoded by the fat facets gene, Science 270 (1995) 1828-1831.

[56] X. Wu, L. Yen, L. Irwin, C. Sweeney, K.L. Carraway III, Stabilization of the E3 ubiquitin ligase Nrdp1 by the deubiquitinating enzyme USP8, Mol. Cell. Biol. 24 (2004) 77487757.

[57] M. Canning, C. Boutell, J. Parkinson, R.D. Everett, A RING finger ubiquitin ligase is protected from autocatalyzed ubiquitination and degradation by binding to ubiquitinspecific protease USP7, J. Biol. Chem. 279 (2004) 3816038168. 